\title{
Plasmid-mediated AmpC $\beta$-lactamase (CMY-2) gene in Salmonella typhimurium isolated from diarrheic pigs in South Korea
}

\author{
Ki-Eun Lee ${ }^{1}$, Seong-In Lim', Hwan-Won Choi², Suk-Kyung Lim', Jae-Young Song ${ }^{1}$ and Dong-Jun An ${ }^{1 *}$
}

\begin{abstract}
s
Background: Salmonella resistant to third-generation cephalosporin has been isolated from an increasing number of animals worldwide. The purpose of this study was to examine ESBL (extended-spectrum $\beta$-lactamases)-producing and PABL (plasmid-mediated AmpC $\beta$-lactamases)-producing Salmonella isolates from pigs in South Korea.

Results: Salmonella Typhimurium KVCC-BA1300259 was resistant to ampicillin, amoxicillin/clavulanic acid, cephalothin, chloramphenicol, florfenicol, cefoxithin, gentamicin, nalidixic acid, trimethoprim/sulfamethoxazole, tetracycline, and ceftiofur. The results of a double-disk synergy test and PCR confirmed that the isolate produced CMY-2 (PABL). Analysis of plasmid incompatibility (Inc) groups revealed the presence of IncA/C and IncFIB, indicating antimicrobial resistance. This study is the first to identify S. Typhimurium isolates harboring CMY-2 in pigs in South Korea.

Conclusions: The presence of CMY-2 in pigs poses a significant threat of possible horizontal spread between animals and humans.
\end{abstract}

Keywords: Salmonella typhimurium, Pig, Plasmid, PABL, CMY-2

\section{Background}

Salmonella sp. are important zoonotic pathogens, are widespread, and can colonize or infect a variety of domesticated and wild animals, including mammals, birds, and reptiles [1-3]. In pigs, Salmonellosis is an infectious digestive disease, which presents with acute or chronic symptoms. Salmonella Choleraesuis and Salmonella Typhimurium are the two main causative agents of salmonellosis worldwide [4]; however, $S$. Typhimurium is the main cause of disease in pigs in Korea [5].

Since cephalosphorin was developed as an antimicrobial agent, an expanded-spectrum cephalosphorin is recommended for the treatment of salmonellosis [6]. However, Salmonella can produce $\beta$-lactamase, which digests thirdgeneration cephalosphorins and renders them ineffective $[7,8]$. Antimicrobial resistance to cephalosphorin is conferred by extended-spectrum $\beta$-lactamases (ESBL) and plasmid-mediated AmpC $\beta$-lactamases (PABL) [9]. ESBL-producing Salmonella isolates produce CTX-M,

\footnotetext{
* Correspondence: andj67@korea.kr

${ }^{1}$ Animal and Plant Quarantine Agency, Anyang, Republic of Korea Full list of author information is available at the end of the article
}

TEM, OXA or SHV-derived ESBL $[6,8,10,11]$. Recently, Salmonella has developed resistance to cephalosporin through the transmission of PABL [12], of which CMY-2 is the most common. CMY-2 was first reported in the USA and is the most widely distributed PABL, with cases also reported in France, Germany, Greece and the United Kingdom; indeed, it was recently isolated from a cow in Japan and from pigs in China [1,3,12-14]. In most cases, the CMY-2 gene is present in large plasmids, of which several genetic types have been reported. Because it is encoded within a plasmid, CMY-2 can be transmitted horizontally. Thus, there is increasing concern that PABL may spread among pathogens circulating in animals and humans [6].

Here, we isolated CMY-2-producing $S$. Typhimurium isolates from diarrheic pigs in South Korea, and examined the potential horizontal transmission of PABL determinants through plasmids.

\section{Methods}

Isolation and identification of Salmonella

Porcine fecal samples were collected from livestock with digestive disease, such as diarrhea and enteritis by 
Choong-Ang Vaccine Laboratories of animal appraisal organization. A total of 483 diarrheal fecal samples were obtained from nine provinces (Gyeonggi, Gangwon, Chungbuk, Chungnam, Jeonbuk, Jeonnam, Gyeongbuk, Gyeongnam, and Jeju) in South Korea from June 2011 to June 2012. Samples were mixed with $45 \mathrm{ml}$ of buffered peptone water and incubated for $20 \mathrm{~h}$ at $37^{\circ} \mathrm{C}$. After incubation, $0.1 \mathrm{ml}$ of sample was inoculated into $10 \mathrm{ml}$ Rappaport Vassiliadis R10 broth (RV, Merck, Germany) and then incubated for $24 \mathrm{~h}$ at $42^{\circ} \mathrm{C}$. One loop of RV culture was streaked onto the surface of XLD agar (Difco, USA) and Salmonella-Shigella agar (Difco) plates and the suspected colonies were serotyped using Salmonella antisera (Denka Seiken, Tokyo, Japan) according to the method of Ewing [4]. Isolates of Salmonella sp. were deposited in the Korea Veterinary Culture Collection (KVCC), where they were stored at $-70^{\circ} \mathrm{C}$ until further use.

\section{Measurement of minimal inhibitory concentrations and double-disk synergy tests}

Minimal inhibitory concentrations (MICs) were determined using the standard broth dilution methods described in Clinical and Laboratory Standard Institute (CLSI) guidelines. Escherichia coli ATCC 25922 was used as a control strain. The double-disk synergy test (DDST), which is used to detect $\beta$-lactamases, was performed with either $30 \mu \mathrm{g}$ cefotaxime or $30 \mu \mathrm{g}$ ceftazidime alone, or with either $30 \mu \mathrm{g}$ cefotaxime or $30 \mu \mathrm{g}$ ceftazidime plus $10 \mu \mathrm{g}$ clavulanic acid according to CLSI guidelines. The DDST was considered positive when the inhibition zone produced by the combined effects of either ceftazidime or cefotaxime plus clavulanic acid was $\geq 5 \mathrm{~mm}$ larger than that produced by either ceftazidime or cefotaxime alone.

\section{Amplification and sequencing of $\beta$-lactamases}

Multiplex PCR to detect ESBL or PABL genes was performed as described previously [1]. DNA fragments were separated on a 1\% agarose gel. Fragments of the appropriate size [1] were extracted from the gel and purified using a Gel Extraction kit (Qiagen Inc., CA, USA), followed by sequencing in an ABI Prism 373 Genetic Analyzer (Applied Biosystems, Foster City, USA) using Sanger's method. A database search was then performed using the BLAST program at NCBI (http://www.ncbi. nlm.nih.gov).

\section{Conjugation testing}

Conjugation with a sodium azide-resistant recipient, Escherichia coli J53, was performed using broth methods [9]. Conjugation strains were selected by plating on MacConkey agar containing $64 \mathrm{mg} / \mathrm{L}$ of ceftazidime and $128 \mathrm{mg} / \mathrm{L}$ of sodium azide.

\section{Plasmid analysis}

Plasmid DNA was purified using a Plasmid mini purification kit (Qiagen Inc., CA, USA). The BAC-Tracker supercoiled DNA ladder (Epicentre Biotechnologies Inc., WI, USA) was used as a size marker for plasmid analysis. Plasmids were analyzed using the PCR-based replicon typing method to identify the plasmid type [15]. All detected replicon types were confirmed by sequencing.

\section{Results}

Forty-four Salmonella sp were isolated from 483 diarrhea samples. Of these, 35 strains were serotyped as Typhimurium (S. Typhimurium). The standard broth dilution method was used to determine the antimicrobial susceptibility of $S$. Typhimurium. The antimicrobial susceptibility of the $35 \mathrm{~S}$. Typhimurium strains is shown in Table 1. Two strains (S. Typhimurium KVCC-BA1300259 and $S$. Typhimurium KVCC-BA1300271) were resistant to ampicillin, amoxicillin/clavulanic acid, cephalothin, chloramphenicol, florfenicol, cefoxithin, gentamicin, nalidixic acid, trimethoprim/sulfamethoxazole, tetracycline, and ceftiofur (data not shown). However, S. Typhimurium KVCCBA1300259 (isolated in the Chungnam region) was positive in the DDST, with the zone of inhibition for ceftazidime plus clavulanic acid being $\geq 5 \mathrm{~mm}$ larger than that for ceftazidime alone. Thus, KVCC-BA1300259 was classified as an ESBL-producer. Moreover, genetic analysis revealed that this isolate produced ESBL and $\beta$-lactamase. PCR with primers specific for CMY-2 amplified an 856 bp DNA fragment. Sequence analysis of the CMY-2 gene revealed $100 \%$ homology with the Salmonella plasmid CMY-2 AmpC beta-lactamase gene (GenBank accession no. JN714983).

Table 1 Antimicrobial susceptibility of Salmonella typhimurium isolated from diarrheic pigs

\begin{tabular}{lccc}
\hline \multirow{2}{*}{ Antimicrobial agent } & \multicolumn{3}{c}{ Salmonella typhimurium ( $\mathbf{n}$ 35) } \\
\cline { 2 - 4 } & $\mathbf{S}^{\mathbf{a}} \mathbf{( \% )}$ & $\mathbf{I}(\mathbf{\%})$ & $\mathbf{R}(\mathbf{\%})$ \\
\hline Ampicillin & 22.9 & - & 77.1 \\
Amoxicillin/clavulanic acid & 88.6 & 5.7 & 5.7 \\
Cephalothin & 68.6 & 17.1 & 14.3 \\
Chloramphenicol & 37.1 & 2.9 & 60.0 \\
Florfenicol & 28.6 & 11.4 & 60.0 \\
Cefoxitin & 88.6 & 5.7 & 5.7 \\
Gentamicin & 28.6 & 2.8 & 68.6 \\
Nalidixic acid & 28.6 & - & 71.4 \\
Streptomycin & & & \\
Trimethoprim/sulfamethoxazole & 65.7 & - & 34.3 \\
Tetracycline & 11.4 & - & 88.6 \\
Ceftiofur & 91.4 & 2.9 & 5.7 \\
\hline
\end{tabular}

${ }^{a}$ S, susceptible; I, intermediate; $R$, resistant.

${ }^{\mathrm{b}}$ No CLSI guidelines. 
Antimicrobial resistance of KVCC-BA1300259 was transferred to recipient $E$. coli $\mathrm{J} 53$ by conjugation. The KVCC-BA1300259-TC (transconjugant) was resistant to chloramphenicol, gentamicin, streptomycin, tetracycline, ampicillin, amoxicillin/clavulanic acid, cefoxitin, ceftiofur, and cephalothin (Table 2). PCR detected CMY-2 genes in both KVCC-BA1300259 and KVCC-BA1300259-TC (transconjugant).

S. Typhimurium KVCC-BA1300259 and KVCC-BA1300259TC harbored a common plasmid ranging from $18 \mathrm{~kb}$ to $25 \mathrm{~kb}$ in size, and PCR-based plasmid typing identified the incompatibility (Inc) type of this plasmid as IncA/C and IncFIB (Table 2).

\section{Discussion}

Ceftiofur, which was developed strictly for veterinary use, is used throughout the world to treat diseased livestock [7]. However, animal infection by ESBL-producing and PABL-producing Salmonella has increased worldwide. It is thought that these bacteria emerged in response to the over-use of ceftiofur $[6,16]$.

One hundred and sixty-five Salmonella sp strains were isolated from cattle in China between 2010 and 2011. Of these, 25 strains harbored $\beta$-lactamases. OXA- 1 was the most commonly identified $\beta$-lactamase gene $(n=14)$, followed by TEM-1 $(n=6)$, PSE-1 $(n=4)$, and CMY-2 $(\mathrm{n}=1)$ [1]. A study of 283 Salmonella sp isolated from Korean chickens between 2002 and 2010 showed that 17 of the ceftiofur-resistant isolates were positive for genes encoding CTX-M-14 and CTX-M-15 [9]. Another study found that two $S$. Typhimurium strains isolated from cattle in Japan harbored both TEM-1 and CMY-2 [14]. Plasmid-mediated AmpC- $\beta$-lactamases are frequently identified in human Salmonella isolates in South Korea [17]; however, until now, CMY-2 has not been isolated from cattle or pigs. The present study is the first to report the isolation of CMY-2-producing $S$. Typhimurium from pigs in South Korea. The potential spread of CMY-2producing $S$. Typhimurium via food, particularly animal- derived foods, has important public health implications because CMY-2 is usually plasmid-encoded.

These plasmids can be classified according to size, composition, and incompatibility (Inc) type, and by plasmid multilocus sequence typing $[12,14,18]$. More recently, the Inc type has been used to classify plasmids. This method is an important tool for tracking the diffusion of plasmids conferring antimicrobial resistance [15]. Of the different Inc types, both IncI1 and IncA/C plasmids were common carriers. The IncI1 plasmid only carries the CMY resistance determinant, whereas the IncA/C plasmids carry at least one additional determinant. The IncA/ $\mathrm{C}$ plasmids carry genes that confer resistance to at least four antimicrobial agents: chloramphenicol, gentamicin, streptomycin, and tetracycline $[12,14]$.

Plasmids can be horizontally transmitted between bacterial populations via conjugation or mobilization. CMY $\beta$-lactamase-encoding plasmids harbored by human Salmonella isolates in the USA tended to be either large MDR IncA/C plasmids or IncI1 plasmids harboring a single resistance determinant [12]. The IncA/C and IncI1 plasmids were the most common CMY-2 replicon type identified in human Salmonella isolates in Spain between 2001 and 2005 [6]. The plasmid replicon types of CMY-2 $\beta$-lactamase-producing $S$. Typhimurium isolated from a cow in Japan were IncI1 and IncA/C [14]. However, the plasmids identified in the present study were IncA/C and IncFIB. IncFIB was a single chimeric plasmid containing more than one replication type.

Adding antimicrobial agents to animal feed was prohibited in South Korea in July 2011. In the light of the new regulations, continuous monitoring of antimicrobial susceptibility in strains isolated from livestock is warranted due to the increasing prevalence of antimicrobial resistance.

\section{Conclusion}

S. Typhimurium isolates from livestock pigs in South Korea harbored CMY-2, implying the potential transfer of antimicrobial resistance. This finding suggests that

Table 2 Minimum inhibitory concentrations, plasmid replicon types, and $\beta$-lactamase genes expressed by Salmonella typhimurium isolated from pigs

\begin{tabular}{|c|c|c|c|c|c|c|c|c|c|c|c|c|c|c|}
\hline \multirow{2}{*}{ Strain } & \multicolumn{12}{|c|}{ Minimum inhibitory concentration $(\mu \mathrm{g} / \mathrm{ml})$} & \multirow{2}{*}{$\begin{array}{c}\text { Plasmid size }(\mathbf{k b}) / \\
\beta \text {-lactamase }\end{array}$} & \multirow{2}{*}{$\begin{array}{l}\text { Replicon } \\
\text { type }\end{array}$} \\
\hline & AMP & AUG & CEP & $\mathrm{CHL}$ & FFN & FOX & GEN & NAL & STR & SXT & TET & $\mathrm{XNL}$ & & \\
\hline $\begin{array}{l}\text { S. typhimurium } \\
\text { KVCC-BA1300259 }\end{array}$ & $>64$ & $>64 / 32$ & $>64$ & $>64$ & $>64$ & $>32$ & $>32$ & $>128$ & $>128$ & $>4 / 76$ & $>128$ & $>8$ & $18-25^{\mathrm{CMY}-2}$ & IncA/C IncFIB \\
\hline $\begin{array}{l}\text { S. typhimurium } \\
\text { KVCC-BA1300259-TC }\end{array}$ & $>64$ & $>64 / 32$ & $>64$ & $>64$ & $>64$ & $>32$ & $>32$ & 4 & $>128$ & $<0.1 / 2.3$ & $>128$ & $>8$ & $18-25^{\mathrm{CMY}-2}$ & IncA/C IncFIB \\
\hline E.coli J53 Azider & 8 & $16 / 8$ & 32 & 4 & $<2$ & 4 & $<1$ & 4 & 8 & $<0.1 / 2.3$ & $<2$ & $<0.5$ & & \\
\hline E.coli ATCC25922 & 4 & $4 / 2$ & 16 & 4 & $<2$ & 2 & $<1$ & $<2$ & 4 & $<0.1 / 2.3$ & $<2$ & $<0.5$ & & \\
\hline
\end{tabular}

AMP: ampicillin; AUG: amoxicillin/clavulanic acid; CEP: cephalothin; CHL: chloramphenicol; FFN: florfenicol; FOX: cefoxitin; GEN: gentamicin; NAL: nalidixic acid; STR: streptomycin; SXT: trimethoprim/sulfamethoxazole; TET: tetracycline; XNL: ceftiofur.

TC: transconjugant.

Azide $^{\text {r: }}$ sodium azide resistant. 
plasmids harboring CMY-2 pose a significant threat of horizontal transmission between animals and humans.

\section{Competing interests}

The authors declare that they have no competing interests.

\section{Authors' contributions}

All authors read and approved the manuscript. All authors contributed to the writing of the paper. KL was primarily responsible for collecting the samples and performing the laboratory tests.

\section{Acknowledgments}

We are grateful to the Choong-Ang Vaccine Laboratories for providing the Salmonella samples.

\section{Author details}

'Animal and Plant Quarantine Agency, Anyang, Republic of Korea.

${ }^{2}$ ChoongAng Vaccine Laboratories, Daejon, Republic of Korea.

Received: 5 February 2014 Accepted: 27 May 2014

Published: 2 June 2014

\section{References}

1. Li R, Lai J, Wang Y, Liu S, Li Y, Liu K, Shen J, Wu C: Prevalence and characterization of Salmonella species isolated from pigs, ducks and chickens in Sichuan Province, China. Int J Food Microbiol 2013, 163:14-18.

2. Ridley A, Threlafall EJ: Molecular epidemiology of antibiotic resistance genes in multiresistant epidemic Salmonella typhimurium DT104. Microb Drug Resist 1998, 4:113-118.

3. Winokur PL, Brueggemann A, DeSalvo DL, Hoffmann L, Apley MD, Uhlenhopp EK, Pfaller MA, Doern GV: Animal and human multidrugresistant, cephalosporin-resistant salmonella isolates expressing a plasmid-mediated CMY-2 AmpC beta-lactamase. Antimicrob Agents Chemother 2000, 44:2777-2783.

4. Ewing WH: Serologic identification of Salmonella. In Edwards and Ewing's Identification of Enterobacteriaceae. 4th edition. Edited by Ewing WH. New York: Elsevier Science Publishing Co., Inc.; 1986:201-238.

5. Lim SK, Lee HS, Nam HM, Jung SC, Koh HB, Roh IS: Antimicrobial resistance and phage types of Salmonella isolates from Healthy and diarrheic pigs in Korea. Foodborne Pathog Dis 2009, 6:981-987.

6. González-Sanz R, Herrera-León S, de la Fuente M, Arroyo M, Escheita MA: Emergence of extened-spectrum $\beta$-lactamases and AmpC-type $\beta$-lactamases in human Salmonella isolated in Spain from 2001 to 2005. J Antimicrob Chemother 2009, 64:1181-1186.

7. Lee KE, Lee YH: Isolation of Multidrug-Resistant Salmonella typhimurium DT104 from swine in Korea. J Microbiol 2007, 45:590-592.

8. Morris D, Whelan M, Corbett-Feeney G, Cormican M, Hawkey P, Li X, Doran G: First report of extended-spectrum-beta lactamase-producing Salmonella enterica isolates in Ireland. Antimicrob Agents Chemother 2006, 50:1608-1609.

9. Kang MS, Kwon YK, Oh JY, Kim MJ, Call DR, An BK, Shin EG, Song EA, Kwon $\mathrm{JH}$ : Evidence for recent acquisition and successful transmission of bla (CTX-M-15) in Salmonella enterica in South Korea. Antimicrob Agents Chemother 2013, 57:2383-2387.

10. Fernandez Vazquez M, Munoz Bellido JL, Garcia Garcia Ml, Garcia-Rodriguez JA: Salmonella enterica serovar Enteritidis producing a TEM-52 beta-lactamase: first report in Spain. Diagn Microbiol Infect 2006, 55:245-246.

11. Tamang MD, Nam HM, Kim TS, Jang GC, Jung SC, Lim SK: Emergence of extended-spectrum beta-lactamase (CTX-M-15 and CTX-M-14)-producing nontyphoid Salmonella with reduced susceptibility to ciprofloxacin among food animals and humans in Korea. J Clin Microbiol 2011, 49:2671-2675.

12. Folster JP, Pecic G, McCullough A, Rickert R, Whichard R: Characterization of blaCMY-encoding plasmids among Salmonella isolated in the united states in 2007. Foodborne Pathog Dis 2007, 8:1289-1294.

13. Philippon A, Arlet G, Jacoby GA: Plasmid-determined AmpC-type beta-lactamases. Antimicrob Agents Chemother 2002, 46:1-11.

14. Sugawara M, Komori J, Kawakami M, Izumiya H, Watanabe H, Akiba M: Molecular and phenotypic characteristics of CMY-2 beta-lactamase- producing Salmonella enterica serovar Typhimurium isolated from cattle in Japan. J Vet Med Sci 2011, 73:345-349.

15. Carattoli A, Bertini A, Villa L, Falbo V, Hopkins KL, Threlfall EJ: Identification of plasmids by PCR-based replicon typing. J Microbiol Methods 2005, 63:219-228.

16. Yoo JS, Byoen J, Yang J, Yoo Jl, Chung GT, Lee YS: High prevalence of extended-spectrum bata-lactamase and plasmid-mediated AmpC beta-lactamase in Enterobacteriaceae isolated from long-term care facilities in Korea. Diagn Microbiol Infect Dis 2010, 67:261-265.

17. Song W, Kim JS, Kim HS, Park MJ, Lee KM: Appearance of Salmonella enterica isolates producing plasmid-mediated AmpC beta-lactamase, CMY-2, in South Korea. Diagn Microbiol Infect Dis 2005, 52:281-284.

18. Accogli M, Fortini D, Giufre M, Graziani C, Dolejska M, Carattoli A, Cerquetti M: Incl1 plasmids associated with the spread of CMY-2, CTX-M-1 and SHV-12 in Escherichia coli of animal and human origin. Clin Microbiol Infect 2013, 19:E238-40.

doi:10.1186/1756-0500-7-329

Cite this article as: Lee et al:: Plasmid-mediated AmpC $\beta$-lactamase (CMY-2) gene in Salmonella typhimurium isolated from diarrheic pigs in South Korea. BMC Research Notes 2014 7:329.

\section{Submit your next manuscript to BioMed Central and take full advantage of:}

- Convenient online submission

- Thorough peer review

- No space constraints or color figure charges

- Immediate publication on acceptance

- Inclusion in PubMed, CAS, Scopus and Google Scholar

- Research which is freely available for redistribution 